\title{
Collective states of even Xe isotopes in IBM+MQRPA
}

\author{
A. D. Efimov ${ }^{1,2, a}$ and V. M. Mikhajlov ${ }^{3}$ \\ ${ }^{1}$ Admiral Makarov State University of Maritime and Inland Shipping, St.-Petersburg, Russia \\ ${ }^{2}$ A. F. loffe Physical Technical Institute, 194021, St.-Petersburg, Russia \\ ${ }^{3}$ V. A. Fock Institute of Physics, St.-Petersburg State University, 198904, St.-Petersburg, Russia
}

\begin{abstract}
A modification of the Quasiparticle Random Phase Approximation (QRPA) with small ground state correlations is suggested. The lowest energy phonon is used as the image of $d$-boson of the Interacting Boson Model 1 (IBM1) and applied to microscopical calculations of the IBM1 parameters. Results are compared with experimental data for $\mathrm{Xe}$ isotopes.
\end{abstract}

The Quasiparticle Random Phase Approximation (QRPA) is one of the widely used methods for description of semimagic and deformed nuclei [1]. Application of this approximation is quite correct in those cases when the Ground State Correlations (GSC) i.e. the distinction between the quasiparticle vacuum (QV) and the RPA-phonon vacuum (PV), exist but are rather small. Quantitatively it means, e.g., that $\Sigma \psi^{2} \gg \Sigma \varphi^{2}, \psi, \varphi$ being amplitudes of the RPA $D_{\mu}$-phonons $(\mu=0, \pm 1, \pm 2)$

$$
D_{\mu}^{+}=\frac{1}{\sqrt{2}} \sum_{\tau=\{n, p\}} \sum_{i, k}\left[\psi_{i k}(\mu) a_{i}^{+} a_{k}^{+}+\varphi_{i k}(\mu) a_{\bar{k}} a_{\bar{i}}\right] .
$$

Futher, we'll work with the QRPA-phonons composed of the Bogoliubov quasiparticles. In Eq. (1), $a_{i}^{+}, a_{k}$ are creation and annihilation quasiparticle operators, $i$ is a single-particle state in a spherical mean field, $\bar{i}$ is a timeconjugated state.

Direct application of QRPA is not justified in the region where the transition takes place from spherical nuclei to deformed ones that is revealed in lowering the energies of the first $2^{+}$-states (e.g., from $1.2 \mathrm{MeV}$ in $\mathrm{Sn}$ isotopes up to $0.32 \mathrm{MeV}$ in $\left.{ }^{120} \mathrm{Xe}\right)$ and at the same time in increasing $B\left(E 2,2_{1}^{+} \rightarrow 0_{\text {g.s. }}^{+}\right)$. Applying the standard QRPA yields the values $\Sigma \varphi^{2}$ smaller then but comparable with $\Sigma \psi^{2}$ that necessitates to modify QRPA conformably.

Several modification of RPA, included the particlehole RPA and QRPA, have been suggested which take into account GSC more consistently [2-8]. The main idea of our modification of QRPA (MQRPA) was formulated many years ago $[9,10]$ and was developed further in $[11,12]$. We construct special equations like RPA for amplitudes $\psi$ and $\varphi$ of the collective quadrupole phonon $D_{\mu}$, Eq. (1), which give very small values of $\Sigma \varphi^{2}$, i.e. with small GSC in the fermion space. However, after mapping the $D$-phonons and PV onto the boson space the boson Hamiltonian proves to be such that its diagonalization

\footnotetext{
ae-mail: efimov98@mail.ru
}

leads to a rather complicated ground state function comprising apart from the boson vacuum (BV) two, three and more quadrupole bosons. Thus, if one returned from the boson space to the fermion one the ground state in this space would turn out to be strongly correlated.

Transition to the boson description is implemented following the Interacting Boson Model (IBM) in its first variant (IBM1 or SU(6)-model) which was first introduced by Janssen et al. [13] and then in another formulation by Arima and Iachello [14]. The IBM1 Hamiltonian, $H_{\mathrm{IBM}}$, contains 6 parameters $\left(\varepsilon_{d}, k_{1}, k_{2}, C_{L}\right)$ :

$$
H_{\mathrm{IBM}}=\varepsilon_{d} \hat{n}_{d}+k_{1} \hat{p}_{1}+k_{2} \hat{p}_{2}+\frac{1}{2} \sum_{L=0,2,4} C_{L} \hat{C}_{L},
$$

where

$$
\begin{array}{ll}
\hat{n}_{d}=\sum_{\mu} d_{\mu}^{+} d_{\mu} ; & \hat{p}_{1}=d^{+} \cdot d^{+} s^{+} s+\text { H.c.; } \\
\hat{p}_{2}=\left(d^{+} d^{+}\right)^{(2)} \cdot d s+\text { H.c.; } & \hat{C}_{L}=\left(d^{+} d^{+}\right)^{(L)} \cdot(d d)^{(L)} .
\end{array}
$$

In Eqs. (2)-(3), $d^{+}, d, s^{+}, s$ are the quadrupole and scalar boson operators. The seventh parameter is $\Omega-$ the maximum $d$-boson number in boson wave functions.

A microscopical way to calculate the IBM1 parameters was suggested in the first work on IBM [13] where quadrupole $d$-bosons were introduced as an image of the most collective two-quasiparticle phonons composed by both neutron and proton pairs (in the IBM2 there are separately neutron and proton bosons [15]). As the image of $d$-bosons we employ the quadrupole phonons of MQRPA with the lowest energy. As shown by IBM2 [16] and our [12] calculations, in order to obtain the realistic values of IBM parameters one has to renormalize them, taking into account the connection of the collective $D$-phonon or $d$-boson space with phonons of higher energies and angular momenta $L \geq 2$. So all calculations here for $\mathrm{Xe}$ isotopes and in Ref. [12] are performed with the renormalized IBM1 parameters. We consider all states inside of the yrast 
band and several ones outside with energies less than the doubled pairing gap, i.e. $\lesssim 3.0 \mathrm{MeV}$, to be collective states which can be described in the $D$-phonon or $d$-boson space. The energy of such state $E$ is formed by the following three constituents [12]:

$$
E=E_{\mathrm{QV}}+E_{\mathrm{PV}}+E_{\mathrm{IBM}}
$$

$E_{\mathrm{QV}}=\left\langle\mathrm{QV}\left|H_{\mathrm{F}}\right| \mathrm{QV}\right\rangle, E_{\mathrm{PV}}=\left\langle\mathrm{PV}\left|: H_{\mathrm{F}}:\right| \mathrm{PV}\right\rangle$, and $E_{\mathrm{IBM}}=$ $\left\langle I_{v}\left|H_{\mathrm{IBM}}\right| I_{v}\right\rangle$. The first of them, $E_{\mathrm{QV}}$, is the energy of QV and $H_{\mathrm{F}}$ is the fermion Hamiltonian. The second term, $E_{\mathrm{PV}}$, is the energy of PV which is determined by the GSC, : $H_{\mathrm{F}}$ : is the same Hamiltonian but the quasiparticle operators in it stand in the normal order. $E_{\mathrm{IBM}}$ is the expectation value of $H_{\mathrm{IBM}}$, Eq. (2), $\left|I_{v}\right\rangle$ being a boson wave function of a state with spin $I$. Index $v$ shows which time this function appears, the larger $v$ the higher energy of the state $\left|I_{v}\right\rangle=\sum_{n} \alpha_{v n}|n\rangle$, where $|n\rangle$ is a function of the $\mathrm{SU}(5)$-basis.

In Refs. $[11,12]$, we suggested a functional $\Phi$. Minimization of $\Phi$ yields all the quantities determining $E$, Eq. (4), i.e. the Bogoliubov parameters $(u, v), D$-phonon amplitudes $(\psi, \varphi)$ and the set of amplitudes $\left\{\alpha_{v n}\right\}$ of boson wave functions. This functional $\Phi$ involves $E$ and several additional conditions.

At first, such quantities as $(u, v),(\psi, \varphi)$ and $\left\{\alpha_{v n}\right\}$ are to be normalized. Secondly, it is supposed in IBM1 that $D_{\mu}^{+}, D_{v}$ and their commutators form SU(6)-algebra. Therefore, $\Omega$ characterizing a symmetric representation of the SU(6)-algebra is an integer. As the definition of $\Omega$ we take

$$
\left\langle\mathrm{PV}\left|\sum_{\kappa}\left[\left[\left[D_{\kappa}, D_{\kappa}^{+}\right], D_{\mu}^{+}\right], D_{\mu}\right]\right| \mathrm{PV}\right\rangle=6 / \Omega .
$$

In the quasiparticle theory, the expectation values of the neutron and proton number operators have to be equal to the corresponding particle numbers in a given nucleus. Therefore, as usual, the particle number operators with the corresponding chemical potentials are introduced in $\Phi$. Thus, the chemical potentials depend on state $\left|I_{v}\right\rangle$ and their alterations contribute to differences between theoretical level energies. To attain a small value of $\sum \varphi_{i k}^{2}$ we introduce into $\Phi$ a special regulator $R$ of GSC.

The regulator $R$ is proportional to the quasiparticle number operator and can fix the quasiparticle number in $\mathrm{PV}$ or the ratio $r$

$$
R=\frac{\xi}{2} \sum_{i, m_{i}} a_{i}^{+} a_{i} ; r=\sum_{i, k} \varphi_{i, k}^{2}(\mu) / \sum_{i, k} \psi_{i, k}^{2}(\mu) .
$$

The appearance of $R$ in $\Phi$ distinguishes our MQRPA from the standard QRPA.

The explicit form of components entering $\Phi$ depends on the method applied to treat the many-body problem. In particular, one can use the Hartree-Fock-Bogoliubov (HFB) method with a realistic effective interaction. In [11, 12], we exploited a rather simplified approach with the spherical Saxon-Woods mean field and residual effective forces. In our calculations, matrices of the monopole particle-particle forces are approximated by the well-known constants $G_{\tau}(\tau=n, p)$ that allow the standard Bogoliubov transformation $(u, v$ are numbers for each single-particle state) to be employed. The particle-hole and particle-particle forces are factorized in a traditional manner as products of matrix elements of the quadrupole single-particle operators. We do not include in calculations the spinexchange and two-particle spin-orbit interactions. They can reduce the quadrupole collectivity and to compensate omitting those forces, our isoscalar strength of the particlehole force is by $10 \%$ lower than the estimation by A. Bohr and B. Mottelson [17]. Since our approach is rather far from the universal self-consistency we admit some variations of the Saxon-Woods potential parameters to reproduce experimental data better.

Thus, the IBM1 parameters are functions of singleparticle energies, matrix elements and strengths of the effective forces and also of quantities subject to determination, i.e., $(u, v),(\psi, \varphi)$. To display the latter $E_{\mathrm{IBM}}$ we represent it as a sum of the IBM1 parameters multiplied by expectation values (EXPV) of the separate terms of $H_{\mathrm{IBM}}$, namely, $\left\langle I_{v}\left|\hat{n}_{d}\right| I_{v}\right\rangle,\left\langle I_{v}\left|\hat{p}_{1}\right| I_{v}\right\rangle$ etc. At calculations of variations of $E_{\mathrm{IBM}}$ with respect to $(u, v),(\psi, \varphi)$ we allow for only terms with $\varepsilon_{d}$ and $k_{1}$, Eq. (2), where renormalizing factors are considered to be invariable. Our calculation show that renormalized parameters $C_{L}$, Eq. (2), practically do not change at small variations of $(u, v)$ and $(\psi, \varphi)$. Variations of parameter $k_{2}$ with respect to $(u, v)$ could influence single-particle energies as the main dependence of $k_{2}$ on $(u, v)$ is contained in factor $\left(u_{i} u_{k}-v_{i} v_{k}\right)$ which is highly sensitive to a mutual arrangement of single-particle levels. However, a change of the spin-orbital potential depth, which we admit in transitional nuclei such as Xe isotopes, produces much stronger effect on level positions. Therefore, we calculate $k_{2}$ but do not consider its variations, i.e. its influence on the level positions.

At the end, the variations of $\Phi$ with respect to $(u, v)$, $(\psi, \varphi)$ give a set of coupled equations. They include mentioned above EXPV which depends on boson amplitudes $\left\{\alpha_{v n}\right\}$. Thus, the self-consistence procedure is demanded: using $(u, v),(\psi, \varphi)$ one calculates the IBM1 parameters, diagonalizes $H_{\mathrm{IBM}}$, finds $\left\{\alpha_{v n}\right\}$, calculates EXPV and returns to equations for $(u, v),(\psi, \varphi)$ and so on until the self-consistent values of all the unknown quantities are obtained.

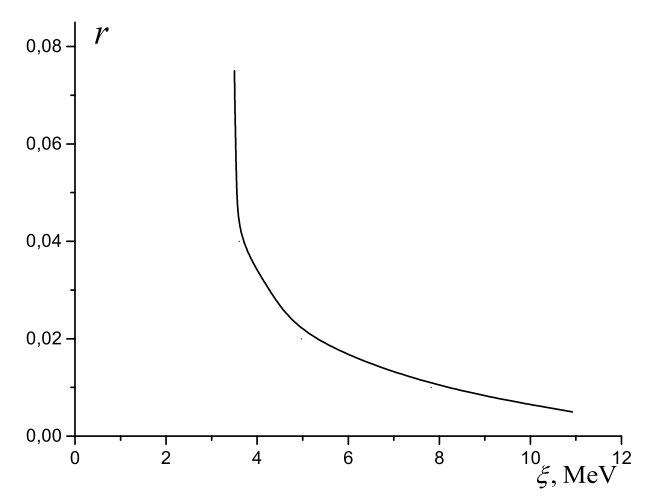

Figure 1. The ratio $r$, Eq. (6), as a function of $\xi$. 


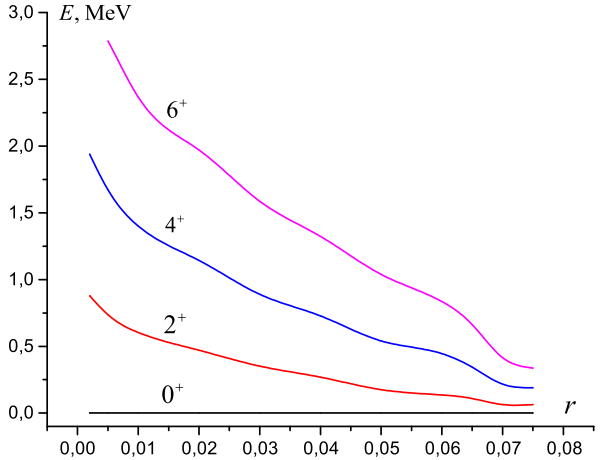

Figure 2. (Color online) The yrast band in ${ }^{124} \mathrm{Xe}$ as a function of $r$ at fixed values of the quadrupole interaction strengthes.

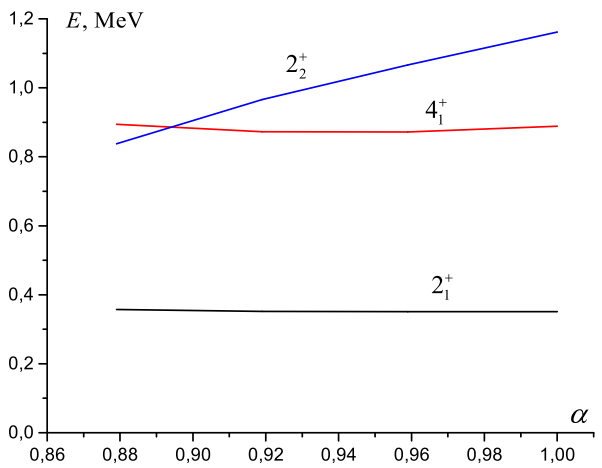

Figure 3. (Color online) Dependence of $E\left(2_{2}^{+}\right), E\left(4_{1}^{+}\right), E\left(2_{1}^{+}\right)$on $\alpha$ for ${ }^{124} \mathrm{Xe}$.

Results following from the equations for $(u, v)$ show that the presence of bosons in the ground and excited states exerts blocking effect on the pairing, i.e. the pairing correlations are attenuated with the growth of spin and excitation energy. Thus, we confirm conclusions of calculations with Tamm-Dankoff phonons in fermion space [18]. However, inclusion the $E_{\mathrm{IBM}}$ value in $(u, v)$-variations at values of $\left\langle I_{v}\left|\hat{n}_{d}\right| I_{v}\right\rangle \geq 2$ (that takes place in Xe isotopes) results in delaying the attenuation caused by the quadrupole particle-hole forces. Thereby neutrons affect on proton pairing and vice versa. The effect of increasing neutron numbers on the proton pairing by means of the particlehole forces is worth notice at analysis of the pairing energies in isotopic chains and, at replacement of protons by neutrons in isotonic ones as well.

Equations for $(\psi, \varphi)$ arising mainly at variations of $E_{\mathrm{IBM}}$ involve the regulator $R$, Eq. (6). Since $R$ joins to two-quasiparticle energies in $\varepsilon_{d}$ and $k_{1}$ it is able to change the character of the phonon collectivity. The more is $\xi$ the further from zero is the first pole in the secular equation for the phonon energy $\omega$ and the smaller are values of corresponding sums at $\omega=0$. This leads to increasing $\omega$

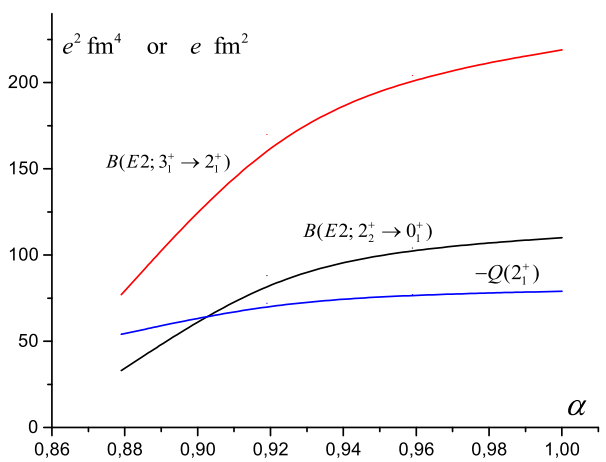

Figure 4. (Color online) Dependence of $Q\left(2_{1}^{+}\right)$(in $e \mathrm{fm}^{2}$ units), $B\left(E 2 ; 2_{2}^{+} \rightarrow 0_{1}^{+}\right)$, and $B\left(E 2 ; 3_{1}^{+} \rightarrow 2_{1}^{+}\right)$(in $e^{2} \mathrm{fm}^{4}$ units ) on $\alpha$ for ${ }^{124} \mathrm{Xe}$.

and, as the result, to a weakening of GSC. Note, that in our approach $\omega$ is always higher than the $d$-boson energy $\varepsilon_{d}$. Figure 1 shows $r$ as a function of $\xi$. The quasiparticle number in $\mathrm{PV}, N_{\mathrm{qp}}$, is practically proportional to $r$ : $N_{\mathrm{qp}} \simeq 12.1 r+0.1$, where $0.02<r<0.08\left(N_{\mathrm{qp}} \rightarrow 0\right.$ at $\left.r \rightarrow 0\right)$. We did not found unique criterion that could give an undoubted value of $N_{\mathrm{qp}}$ or $r$. However, there are several arguments narrowing the region of their search. Firstly, it is the fulfilment of the exclusion principle. Addition of $\xi$ to each two-quasiparticle energy draws together and equalizes $D$-phonon amplitudes. Thereby the quasiparticle number $n_{j}$ in each single-particle $j$-state (given by one phonon) decreases. Therefore, even at large enough boson number in a state $\left|I_{v}\right\rangle$ the $n_{j}$ value can remain $<j+1 / 2$. However, it is possible only at the value of $\xi \geq 5 \mathrm{MeV}$ and $r<0.075$ (Fig. 1). These numbers are found in calculations for Xe isotopes. Secondly, to achieve the selfconsistency between $(u, v),(\psi, \varphi)$ and $\left\{\alpha_{v n}\right\}$, mentioned above, the values of $\xi$ and $r$ have to be larger than $5 \mathrm{MeV}$ and less than 0.075, respectively. Thirdly, as shown in [5], a more correct boson mapping of the $D$-phonon operators, to get SU(6)-algebra, can be attained at small values of $\sum_{i k} \varphi_{i k}^{2}=0.05 \approx r$. If the effective interaction strengths were not subject to small alterations to fit them in with experimental spectra, then we could choose the value of $\xi$ to reproduce better the experimental data as at fixed strengths the yrast spectrum is compressed with increasing $r$ that is shown in Fig. 2.

All our calculations for $\mathrm{Xe}$ isotopes are carried out with $r \sim 0.02 \div 0.03(\xi \simeq 5 \mathrm{MeV})$ (see Fig. 1). These values of $r$ and $\xi$ ensure the fast convergence of the selfconsistency process and fulfilment of the exclusion principle. This value of $\xi$ gives rise to the extension of the $D$-phonon space, i.e. to increasing the maximum $d$-boson number $\Omega$ because of increasing a relative role of the high energy two-quasiparticle states in the $D$-phonon structure.

Apart from the interaction strengths in the fermion Hamiltonian we vary the spin-orbital potential depth $V_{l s}$ 


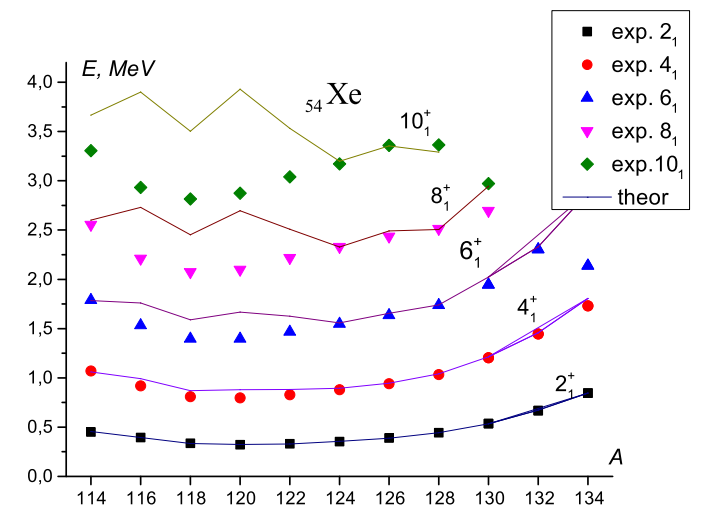

Figure 5. (Color online) Theoretical and experimental energies of the yrast states in Xe-isotopes.

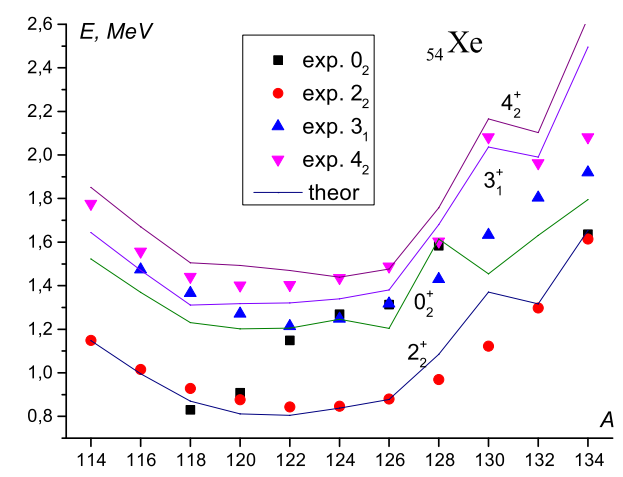

Figure 6. (Color online) Theoretical and experimental energies of non-yrast states in Xe-isotopes.

which strongly affects the value of parameter $k_{2}$ in $H_{\mathrm{IBM}}$, Eq. (2). We keep invariable all parameters of the spinorbital potential taken from Ref. [19] (Table 4, set 1) with the only exception of the depth. We denote the depth as $\alpha V_{l s}\left(V_{l s}\right.$ is given in [19]) and slightly change parameter $\alpha(0.8<\alpha<1)$. At a fixed IBM parameter $\varepsilon_{d}$ the growth of $k_{2}$ (it almost linearly follows $\alpha$ ) is accompanied by convergence of the yrast band states and rising the energies of $2_{2}^{+}-, 3_{1}^{+}$-collective states simultaneously with probabilities of inter-band E2-transitions (e.g., $2_{2}^{+} \rightarrow 0_{1}^{+}$). To compensate the impact of $\alpha$ (through $k_{2}$ ) on the yrast state energies we alter linearly with $\alpha$ the quadrupole strengths but not more than by $3 \%$. The dependencies on $\alpha$ of state energies, E2-probabilities and the quadrupole moment of the $2_{1}^{+}$-state are given in Figs. 3 and 4 . Thus, the value of $\alpha$ can be fixed for each of Xe isotopes and more or less reasonable agreement with experimental data can be obtained. We did not investigate in detail the influence of the effective forces entering into parameters of $H_{\text {IBM }}$ on single-particle energies and so we cannot assert that the weakening of the spin-orbital potential $\left(\alpha_{\text {average }} \simeq 0.9\right)$ cannot be imitated by this influence. However, it is undoubted that the presence of the collective quadrupole phonons (bosons) could change the spatial density on the nuclear edge and, as $V_{l s} \sim \partial \rho / \partial r$, affects the spin-orbital potential.
Table 1. $B(E 2)$ (in $e^{2} \cdot \mathrm{fm}^{4}$ units) inside the yrast bands in Xe isotopes.

\begin{tabular}{ccccc}
\hline \multicolumn{3}{c}{${ }^{116} \mathrm{Xe}$} & \multicolumn{2}{c}{${ }^{120} \mathrm{Xe}$} \\
$I_{i}^{+} \rightarrow I_{f}^{+}$ & exp. & theor. & exp. & theor. \\
\hline $2_{1}^{+} \rightarrow 0_{1}$ & $2470 \pm 92$ & 2460 & $3560 \pm 160$ & 3600 \\
$4_{1}^{+} \rightarrow 2_{1}$ & $4320 \pm 190$ & 3680 & $4120 \pm 300$ & 5290 \\
$6_{1}^{+} \rightarrow 4_{1}$ & $3890_{-300}^{+360}$ & 4330 & $5100_{-1230}^{+1240}$ & 6100 \\
$8_{1}^{+} \rightarrow 6_{1}$ & $3370_{-360}^{+450}$ & 4770 & $3650_{-580}^{+850}$ & 6590 \\
$10_{1}^{+} \rightarrow 8_{1}$ & $3830_{-600}^{+870}$ & 5070 & $3230_{-460}^{+630}$ & 6900 \\
\hline \multicolumn{5}{c}{$122 \mathrm{Xe}$} \\
$I_{i}^{+} \rightarrow I_{f}^{+}$ & exp. & theor. & exp. & theor. \\
\hline $2_{1}^{+} \rightarrow 0_{1}^{+}$ & $2890 \pm 120$ & 2870 & $1560 \pm 85$ & 1580 \\
$4_{1}^{+} \rightarrow 2_{1}^{+}$ & $4150 \pm 190$ & 4250 & $2270 \pm 200$ & 2390 \\
$6_{1}^{+} \rightarrow 4_{1}^{+}$ & $3820_{-1010}^{+2120}$ & 4970 & $2990_{-250}^{+300}$ & 2860 \\
$8_{1}^{+} \rightarrow 6_{1}^{+}$ & $2980_{-1000}^{+3000}$ & 5450 & $3680_{-360}^{+450}$ & 3190 \\
$10_{1}^{+} \rightarrow 8_{1}^{+}$ & $4450_{-1300}^{+310}$ & 5820 & - & - \\
\hline
\end{tabular}

Table 2. $B(E 2)$ (in $e^{2} \cdot \mathrm{fm}^{4}$ units) and $Q\left(2_{1}^{+}\right)$(in $e \cdot \mathrm{fm}^{2}$ units) in ${ }^{124} \mathrm{Xe}$.

\begin{tabular}{cccccc}
\hline$I_{i}^{+} \rightarrow I_{f}^{+}$ & exp. & theor. & $I_{i}^{+} \rightarrow I_{f}^{+}$ & exp. & theor. \\
\hline $2_{1}^{+} \rightarrow 0_{1}^{+}$ & $2120 \pm 54$ & 2150 & $3_{1}^{+} \rightarrow 2_{1}^{+}$ & $84 \pm 15$ & 77 \\
$4_{1}^{+} \rightarrow 2_{1}^{+}$ & $2490 \pm 70$ & 3130 & $3_{1}^{+} \rightarrow 4_{1}^{+}$ & $925 \pm 200$ & 870 \\
$6_{1}^{+} \rightarrow 4_{1}^{+}$ & $3250_{-260}^{+310}$ & 3630 & $5_{1}^{+} \rightarrow 3_{1}^{+}$ & $1380 \pm 190$ & 2180 \\
$2_{2}^{+} \rightarrow 2_{1}^{+}$ & $1140_{-450}^{+2150}$ & 2210 & $5_{1}^{+} \rightarrow 4_{2}^{+}$ & $1310 \pm 250$ & 1020 \\
$2_{2}^{+} \rightarrow 0_{1}^{+}$ & $28_{-7}^{+9}$ & 33 & $5_{1}^{+} \rightarrow 4_{1}^{+}$ & $27 \pm 5$ & 27 \\
$3_{1}^{+} \rightarrow 2_{2}^{+}$ & $3020 \pm 540$ & 2640 & $Q\left(2_{1}^{+}\right)$ & - & -54 \\
\hline
\end{tabular}

Figures 5 and 6 compare the theoretical energies of the yrast band states and of states $2_{2}^{+}, 0_{2}^{+} 3_{1}^{+}, 4_{2}^{+}$with the experimental data in Xe isotopes. Calculations were performed with $\Omega$ which are about two times higher than the half of the number of valence particles or holes, that is caused by the choice of $\xi$. The levels $2_{1}^{+}, 4_{1}^{+}$in all considered isotopes are in the best agreement with experiment. For ${ }^{124,126} \mathrm{Xe}$ the very good agreement is observed even up to $I=12$. However, as a rule, the theoretical yrast spectra are stretched. Perhaps that explicit consideration of noncollective two-quasiparticle high-spin states $(I \geq 6)$, as it was done in [10], improve the situation. Reasonable description is obtained for $2_{2}^{+}-, 4_{2}^{+}$-states. Apparently for adequate description of the low-lying $0_{2}^{+}$-states in ${ }^{118,120} \mathrm{Xe}$ one has to consider explicitly monopole pair-vibrational states. The better description of $3_{1}^{+}$-states can be obtained by means of the terms of $H_{\mathrm{IBM}}$ containing $6 d$-boson operators (i.e. with taking into account "nonaxiality"). Tables 1 and 2 show that $B(E 2)$ values inside the yrast band and those for inter-band transitions in ${ }^{124} \mathrm{Xe}$ are in reasonable agreement with experimental data.

\section{References}

[1] P. Ring and P. Schuck, The Nuclear Many-Body Problem (Springer, Berlin, 1980)

[2] K. Hara, Prog. Theor. Phys. 32, 88 (1964) 
[3] D.J. Rowe, Rev. Mod. Phys. 40, 153 (1968)

[4] P. Schuck and S. Ethofer, Nucl. Phys. A 212, 269 (1973)

[5] K. Takada, K. Yamada, H. Tsukuma, Nucl. Phys. A 496, 224 (1989)

[6] J. Dukelsky and P. Schuck, Phys. Lett. B 464, 164 (1999)

[7] N. Dinh Dang and V. Zelevinsky, Phys. Rev. C 64, 064319 (2001)

[8] V.E. Mitroshin, Phys. Part. Nucl. 33, 719 (2002)

[9] A.D. Efimov and V.M. Mikhajlov, Bull. Russ. Acad. Sci. Phys. 56, 96 (1992); 61, 522 (1997); 62, 735 (1998); 64, 667 (2000)

[10] A.D. Efimov and V.M. Mikhajlov, Phys. Rev. C 59, 3153 (1999)

[11] A.D. Efimov and V.M. Mikhajlov, Proceedings of the Intern. Conference on Nuclear Structure and Related Topics (NSRT09), eds. A.I. Vdovin, V.V. Voronov,
R.V. Jolos (JINR, Dubna, 2009), vol. 2, 251.

[12] A.D. Efimov and V.M. Mikhajlov, Bull. Russ. Acad. Sci. Phys. 74, 547 (2010); 75, 890 (2011); 76, 857 (2012); 77, 862 (2013)

[13] D. Janssen, R.V. Jolos, F. Dönau, Nucl. Phys. A 224, 93 (1974)

[14] A. Arima and F. Iachello, Phys. Rev. Lett. 35, 1069 (1975)

[15] A. Arima, T. Ohtsuka, F. Iachello, I. Talmi, Phys. Lett. B 66, 205 (1977)

[16] A. Van Egmond and K. Allaart, Nucl. Phys. A 425, 275 (1984)

[17] A. Bohr and B. Mottelson, Nuclear Structure, vol. 2: Nuclear Deformations (Benjamin, New York, 1975)

[18] A.K. Vlasnikov and V.M. Mikhajlov, Bull. Russ. Acad. Sci. Phys. 56, 1738 (1992)

[19] V.I. Isakov, K.I. Erokhina, H. Mach, M. SanchezVega, B. Fogelberg, Eur. Phys. J. A 14, 29 (2002) 